Correspondence

Patrick C.Y. Woo

pcywoo@hku.hk

Received 2 December 2014

Accepted 2 June 2015

\section{Report of isolation of Cryptobacterium curtum from a pelvic abscess}

\author{
Siddharth Sridhar, ${ }^{1}$ Samson S.Y. Wong, ${ }^{1,2,3}$ Emily W.T. Tam, ${ }^{1}$ Susanna \\ K.P. Lau ${ }^{1,2,3}$ and Patrick C.Y. Woo ${ }^{1,2,3}$ \\ ${ }^{1}$ Department of Microbiology, University of Hong Kong, University Pathology Building, Queen Mary \\ Hospital, Hong Kong SAR, PR China \\ ${ }^{2}$ State Key Laboratory of Emerging Infectious Diseases, University of Hong Kong, University \\ Pathology Building, Queen Mary Hospital, Hong Kong SAR, PR China \\ ${ }^{3}$ Research Centre of Infection and Immunology, University of Hong Kong, University Pathology \\ Building, Queen Mary Hospital, Hong Kong SAR, PR China
}

Introduction: To the best of our knowledge, this is the first reported case of isolation of Cryptobacterium curtum from an infected site outside the oral cavity.

Case presentation: We report a patient who presented with fever and abdominal pain following an endoscopic procedure. Imaging confirmed a retroperitoneal abscess secondary to duodenal perforation complicating the endoscopic procedure. Surgical drainage of the abscess was carried out. Microbiological analysis of the pus revealed, among other bacteria, an anaerobic Gram-positive rod-shaped bacterium that was definitively identified as Cryptobacterium curtum by $16 \mathrm{~S}$ rRNA gene sequencing. The patient improved after multiple episodes of retroperitoneal lavage and a prolonged course of antibiotics.

Conclusion: This case demonstrates that C. curtum can be isolated outside the oral cavity in polymicrobial infected sites. The pathogenic significance of this organism in contributing to pyogenic infection requires further study.

Keywords: Cryptobacterium curtum; pelvic abscess; opportunistic infection.

\section{Introduction}

Cryptobacterium curtum is an anaerobic Gram-positive rod found in the oral cavity (Nakazawa et al., 1999). It has occasionally been associated with polymicrobial dental infections such as chronic periodontitis (Downes et al., 2001). Here, we report a case of C. curtum isolation from a polymicrobial retroperitoneal collection complicating iatrogenic duodenal perforation in a young man. To the best of our knowledge, this is the first case of isolation of C. curtum from a non-oral site and highlights the pathogenic potential of this organism. Furthermore, we illustrate the utility of $16 \mathrm{~S}$ rRNA gene sequencing in identifying this uncommonly encountered bacterium, which is prone to misidentification by routine bacteriological identification tests. We also report the antibiotic susceptibility pattern for this organism.

Abbreviations: ALT, alanine aminotransferase; CT, computed tomography; ERCP, endoscopic retrograde cholangiopancreatography; MALDI-TOF MS, matrix-assisted laser desorption/ionization time-offlight mass spectrometry.

\section{Case report}

A 39-year-old Chinese man was admitted with epigastric pain radiating to the back. He was a smoker with a background history of urinary tract stones. On admission, he had no fever with no evidence of peritonism on abdominal examination. Blood tests on admission revealed mild leukocytosis $\left(10.8 \times 10^{9} \mathrm{l}^{-1}\right)$, elevated alanine aminotransferase (ALT; $\left.240 \mathrm{U} \mathrm{l}^{-1}\right)$ and elevated amylase $\left(667 \mathrm{U} \mathrm{l}^{-1}\right)$. Alkaline phosphatase and bilirubin were normal. An ultrasonographic scan of the abdomen showed gall bladder stones with no ductal dilatation. In view of the symptomatology, elevated amylase and ultrasonographic findings, he was diagnosed as having gallstone-associated acute pancreatitis. Over the next few days, the patient achieved good symptomatic relief with analgesia. His leukocyte count, ALT and amylase levels normalized spontan-eously.

Elective laparoscopic cholecystectomy was performed 1 week after admission. Intraoperative cholangiography demonstrated an abrupt cut-off at the lower common bile duct. As intraoperative ductal clearance was not technically feasible, papillotomy via endoscopic retrograde 
cholangiopancreatography (ERCP) was performed the next day. The patient developed acute epigastric pain shortly after the procedure. An intravenous contrast-enhanced computed tomography (CT) scan of the abdomen and pelvis showed a suspicious defect at the posterior wall of the second part of the duodenum associated with extensive pneumoretroperitoneum suggestive of post-ERCP duodenal perforation. Urgent laparotomy revealed a moderate amount of brownish retroperitoneal fluid behind the duodenum tracking down to the retrocaecal region. Drainage and irrigation of the retroperitoneal region was performed. Methylene blue ingestion did not reveal the exact site of duodenal perforation. He received intravenous ticarcillin-clavulanate $(3.2 \mathrm{~g}$ every $8 \mathrm{~h}$ ) and cotrimoxazole ( $960 \mathrm{mg}$ every $12 \mathrm{~h}$ ) based on isolation of Stenotrophomonas maltophilia from one blood culture. Bacterial cultures of intraoperative and drain fluid were negative. There was clinical improvement with clearance of blood cultures, a downward trend of leukocytosis and partial resolution of collections on reassessment CT scans. He was discharged after 5 weeks of treatment.

Two weeks later, he developed abdominal pain and fever $\left(39.4{ }^{\circ} \mathrm{C}\right)$ requiring readmission. Blood tests revealed leukocytosis $\left(13.1 \times 10^{9} 1^{-1}\right)$ and neutrophilia $\left(11.2 \times 10^{9} 1^{-1}\right)$. Liver and renal function tests were within the normal range. Reassessment CT scans showed progression of the retroperitoneal collections with extension to the paravertebral muscles. Subsequent laparotomy revealed frank pus in the retropubic, mesorectal, retrohepatic and bilateral paracolic spaces; furthermore, two discrete $5 \mathrm{~mm}$ perforations were now identified at the posterior wall of the second part of the duodenum and confirmed by active methylene blue extravasation. Duodenal repair was carried out, followed by abdominal closure with silastic mesh.

A Gram stain of intraoperative swabs obtained from the pelvic collection showed leukocytes and Gram-positive cocci. After 1 day of incubation, the aerobic cultures yielded a viridans streptococcus and Haemophilus parahaemolyticus. After 5 days of incubation, the anaerobic culture plate yielded small, non-haemolytic colonies of short Grampositive bacilli (strain PW3128).

Due to the extensive retroperitoneal collections, the patient required six more episodes of lavage, irrigation and drainage through temporary abdominal wall silastic mesh closure. Intraoperative wound swabs and peritoneal lavage fluid specimens taken in subsequent laparotomies yielded bacteria including Acinetobacter baumannii, Pseudomonas aeruginosa, extended spectrum $\beta$-lactamase-producing Klebsiella sp., Enterococcus sp. and Enterobacter sp. Candida albicans was also isolated. Strain PW3128 was no longer found in specimens from subsequent laparotomy. The patient was given intravenous meropenem, metronidazole and fluconazole over the course of hospitalization. Abdominal closure was achieved 5 months after admission and imaging showed resolution of the retroperitoneal collections.

\section{Investigations}

\section{Microbiology}

Strain PW3128 is a Gram-positive, non-spore-forming, non-motile bacillus, which formed small non-haemolytic colonies after incubation in haemin/vitamin Ksupplemented blood agar at $35^{\circ} \mathrm{C}$ under anaerobic conditions (hydrogen gas mixture and palladium catalyst, Concept 1000; Ruskinn). It failed to grow on horse blood agar under aerobic conditions $\left(35^{\circ} \mathrm{C}, 5 \% \mathrm{CO}_{2}\right)$, confirming that the organism was an obligate anaerobe. The organism was catalase negative and indole negative. Older colonies were noted to decolorize when Gram stained, staining Gram-negative. Using the Vitek 2 system (bioMérieux), it was identified as Eggerthella lenta (95\% probability). Using matrix-assisted laser desorption/ionization time-of-flight mass spectrometry (MALDI-TOF MS; Bruker), no reliable identification could be obtained.

\section{5 rRNA gene sequencing and phylogenetic characterization}

The bacterium (strain PW3128) was subjected to DNA extraction, PCR amplification and 16S rRNA gene sequencing as described in our previous publications for anaerobic Gram-positive bacilli (Lau et al., 2004a, b, 2006; Chan et al., 2010). LPW57 5'-AGTTTGATCCTGGCTCAG-3' and LPW205 5' -CTTGTTACGACTTCACCC-3') (Sigma) were used as the PCR and sequencing primers. The sequences of the PCR products were compared with known $16 \mathrm{~S}$ rRNA gene sequences in GenBank by multiple sequence alignment using MUSCLE v.3.8 (Edgar, 2004). Poorly aligned or divergent regions of the aligned DNA sequences were removed using Gblocks v.0.91b with relaxed parameters (Castresana, 2000; Talavera \& Castresana, 2007). Tests for substitution model and phylogenetic tree reconstruction, by the maximum-likelihood method, were performed using MEGA v.5.0.5 (Tamura et al., 2011). A total of $1339 \mathrm{nt}$ (nt 66-1444) of the 16S rRNA gene PCR product was used for multiple alignment and phylogenetic analysis. PCR of the 16S rRNA gene of the isolate showed a band at about $1400 \mathrm{bp}$. There were no base differences and one base difference $(0.07 \%)$ between the 16S rRNA gene sequence of the isolate and that of C. curtum strain DSM 15641 (GenBank accession no. NR074425) and C. curtum strain F0066 (GenBank accession no. GQ422741), respectively, and 122 base differences $(9.1 \%)$ between the $16 \mathrm{~S}$ rRNA gene sequence of the isolate and that of Slackia heliotrinireducens (GenBank accession no. NR074439), indicating that the isolate was a strain of C. curtum (Fig. 1).

\section{Antimicrobial susceptibility testing}

Antimicrobial susceptibility to penicillin, metronidazole and vancomycin was determined by an E-test assay (bioMérieux) on Mueller-Hinton agar. The MICs of penicillin, 


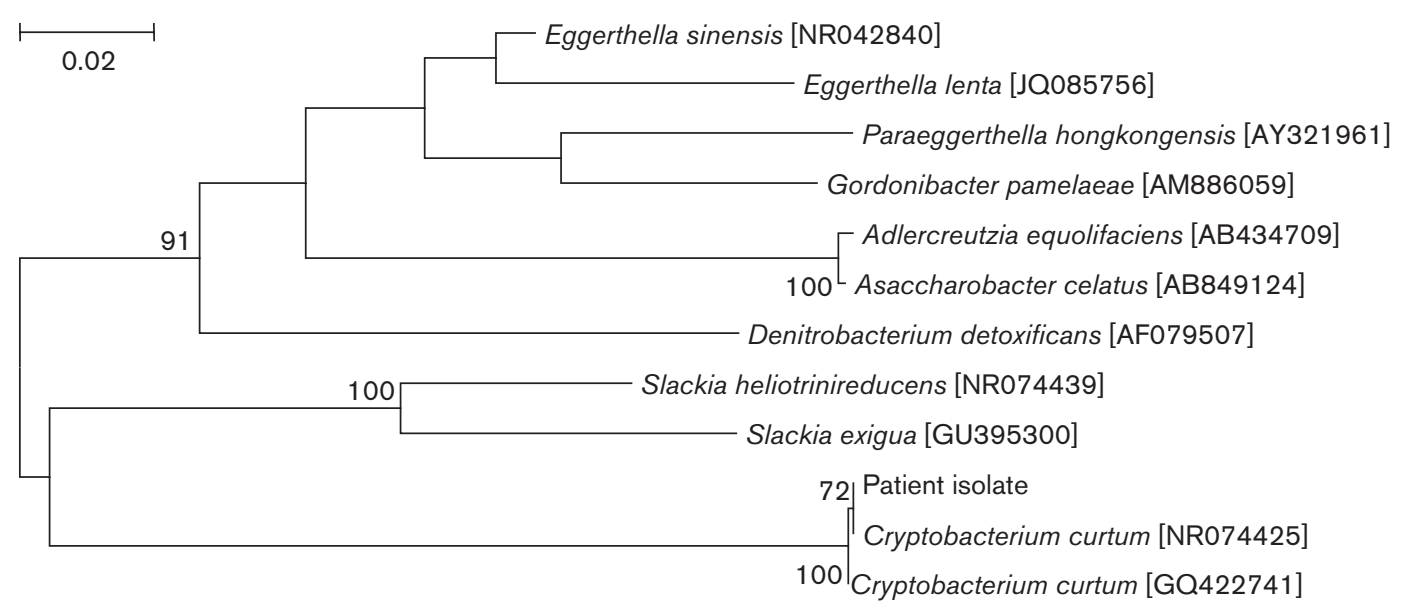

Fig. 1. Phylogenetic analysis of the $16 \mathrm{~S}$ rRNA gene of the patient isolate compared with other closely related species. The tree was reconstructed by the maximum-likelihood method with 1000 bootstrap replicates. Only bootstrap values $\geqslant 60 \%$ are shown. Bar, estimated number of nucleotide substitutions per $50 \mathrm{nt}$. Names and accession numbers are given as cited in GenBank.

metronidazole and vancomycin were $0.16 \mu \mathrm{g} \mathrm{ml}^{-1}$ (susceptible), $0.25 \mu \mathrm{g} \mathrm{ml}^{-1}$ (susceptible) and $1.5 \mu \mathrm{g} \mathrm{ml}^{-1}$, respectively.

\section{Discussion}

We have described a patient with concealed duodenal perforation following an endoscopic procedure complicated by polymicrobial retroperitoneal pelvic collections with C. curtum isolated from pelvic abscess specimens. First described in 1999, C. curtum is an anaerobic, non-sporeforming, Gram-positive bacillus belonging to the phylum Actinobacteria. C. curtum forms a part of the human oral flora and has been implicated as an opportunistic pathogen in dental abscesses and chronic periodontitis, where it is found together with other oral microflora, producing polymicrobial infections (Nakazawa et al., 1999; Downes et al., 2001; Kumar et al., 2003; Mavrommatis et al., 2009; Robertson \& Smith, 2009). This report represents the first description, to the best of our knowledge, of the isolation of C. curtum from an infected site outside the oral cavity.

Besides being a colonizer of the oral cavity, C. curtum may be part of the duodenal microflora. Our patient sustained a perforation of the second descending part of the duodenum complicating an ERCP procedure. The descending duodenum is immobile and retroperitoneal in location. As C. curtum is normally found in the oral cavity, it was likely to be present in oropharyngeal secretions that were swallowed by the patient and subsequently seeded his retroperitoneal space via the descending duodenal perforation. The concomitant isolation of viridans group streptococci and $H$. parahaemolyticus from the retroperitoneal collections also indicated that this was the likely route of infection at this site. This hypothesis is further supported by research showing that the microflora of the proximal small intestine originate predominantly from the oral cavity and throat (Sullivan et al., 2003).

The discontinuation of antibiotics in the presence of an unhealed duodenal perforation was likely to be the most significant factor leading to the development of retroperitoneal collections by antibiotic sensitive oral flora in this patient. Pharmacological factors leading to altered gastric motility and reduced acid production may also favour the survival of oral flora in the upper gastrointestinal tract. Our patient had received inpatient analgesia during the initial hospitalization episode, which was achieved using pethidine, a drug that has been demonstrated to affect gastric motility as well as increase gastric $\mathrm{pH}$ (Schurizek et al., 1989). However, he did not receive any acid suppressive therapy after sustaining the duodenal perforation. Pain relief after the first laparotomy was achieved using tramadol, which has not been shown to modify gastric emptying rates (Maurer et al., 1996; Murphy et al., 1997).

Identification of C. curtum in the clinical microbiology laboratory is difficult. It forms small, translucent, nonhaemolytic colonies on blood agar after prolonged incubation in anaerobic conditions. The rods are very short and stain Gram-positive, although decolorization may be noted in bacteria from older cultures. It is unreactive in many conventional biochemical tests. The Vitek 2 system (bioMérieux) used in this case gave an erroneous identification of Eggerthella lenta. Formal evaluations of the performance of anaerobic commercial identification systems for C. curtum are lacking, and it is likely that many currently available commercial identification kits for anaerobes do not include C. curtum in their database. In our case, MALDI- 
TOF MS also could not produce a reliable identification, reflecting the need for expansion of commercial databases to include reference strains of infrequently seen organisms such as C. curtum (Lau et al., 2012; Tang et al., 2013). Therefore, definitive identification of C. curtum is difficult without $16 \mathrm{~S}$ rRNA gene sequence analysis.

There is a lack of published data on antibiotic sensitivity patterns of C. curtum strains. However, our C. curtum isolate showed in vitro susceptibility to penicillin, vancomycin and metronidazole. This suggests that most commonly used antibiotic combinations for polymicrobial intraabdominal or retroperitoneal collections would be effective against C. curtum.

In summary, we showed for the first time that C. curtum, an anaerobic resident of the oral cavity, may be isolated from extra-oral sites of polymicrobial pyogenic infection. We demonstrated that identification of the organism in clinical microbiology laboratories can be difficult without $16 \mathrm{~S}$ rRNA gene sequencing. The role of this organism in the pathogenesis of pyogenic infection requires further study.

\section{Acknowledgements}

The authors declare no conflicts of interest.

\section{References}

Castresana, J. (2000). Selection of conserved blocks from multiple alignments for their use in phylogenetic analysis. Mol Biol Evol 17, 540-552.

Chan, J. F., Lau, S. K., Woo, P. C., Fan, R. Y., Ip, J. J., Chan, C. F., Luk, J. K. \& Yuen, K. Y. (2010). Lactobacillus rhamnosus hepatic abscess associated with Mirizzi syndrome: a case report and review of the literature. Diagn Microbiol Infect Dis 66, 94-97.

Downes, J., Munson, M. A., Spratt, D. A., Kononen, E., Tarkka, E., Jousimies-Somer, H. \& Wade, W. G. (2001). Characterisation of Eubacterium-like strains isolated from oral infections. $J$ Med Microbiol 50, 947-951.

Edgar, R. C. (2004). MUSCLE: multiple sequence alignment with high accuracy and high throughput. Nucleic Acids Res 32, 1792-1797.

Kumar, P. S., Griffen, A. L., Barton, J. A., Paster, B. J., Moeschberger, M. L. \& Leys, E. J. (2003). New bacterial species associated with chronic periodontitis. J Dent Res 82, 338-344.

Lau, S. K., Woo, P. C., Fung, A. M., Chan, K. M., Woo, G. K. \& Yuen, K. Y. (2004a). Anaerobic, non-sporulating, Gram-positive bacilli bacteraemia characterized by $16 \mathrm{~S}$ rRNA gene sequencing. $J$ Med Microbiol 53, 1247-1253.
Lau, S. K., Woo, P. C., Woo, G. K., Fung, A. M., Wong, M. K., Chan, K. M., Tam, D. M. \& Yuen, K. Y. (2004b). Eggerthella hongkongensis sp. nov. and Eggerthella sinensis sp. nov., two novel Eggerthella species, account for half of the cases of Eggerthella bacteremia. Diagn Microbiol Infect Dis 49, 255-263.

Lau, S. K., Ng, K. H., Woo, P. C., Yip, K. T., Fung, A. M., Woo, G. K., Chan, K. M., Que, T. L. \& Yuen, K. Y. (2006). Usefulness of the MicroSeq 500 16S rDNA bacterial identification system for identification of anaerobic Gram positive bacilli isolated from blood cultures. J Clin Pathol 59, 219-222.

Lau, S. K., Tang, B. S., Curreem, S. O., Chan, T. M., Martelli, P., Tse, C. W., Wu, A. K., Yuen, K. Y. \& Woo, P. C. (2012). Matrix-assisted laser desorption ionization-time of flight mass spectrometry for rapid identification of Burkholderia pseudomallei: importance of expanding databases with pathogens endemic to different localities. J Clin Microbiol 50, 3142-3143.

Maurer, A. H., Krevsky, B., Knight, L. C. \& Brown, K. (1996). Opioid and opioid-like drug effects on whole-gut transit measured by scintigraphy. J Nucl Med 37, 818-822.

Mavrommatis, K., Pukall, R., Rohde, C., Chen, F., Sims, D., Brettin, T., Kuske, C., Detter, J. C., Han, C. \& other authors (2009). Complete genome sequence of Cryptobacterium curtum type strain (12-3). Stand Genomic Sci 1, 93-100.

Murphy, D. B., Sutton, A., Prescott, L. F. \& Murphy, M. B. (1997). A comparison of the effects of tramadol and morphine on gastric emptying in man. Anaesthesia 52, 1224-1229.

Nakazawa, F., Poco, S. E., Ikeda, T., Sato, M., Kalfas, S., Sundqvist, G. \& Hoshino, E. (1999). Cryptobacterium curtum gen. nov., sp. nov., a new genus of Gram-positive anaerobic rod isolated from human oral cavities. Int J Syst Bacteriol 49, 1193-1200.

Robertson, D. \& Smith, A. J. (2009). The microbiology of the acute dental abscess. J Med Microbiol 58, 155-162.

Schurizek, B. A., Willacy, L. H., Kraglund, K., Andreasen, F. \& Juhl, B. (1989). Antroduodenal motility, $\mathrm{pH}$ and gastric emptying during balanced anaesthesia: comparison of pethidine and fentanyl. $\mathrm{Br} J$ Anaesth 62, 674-682.

Sullivan, A., Törnblom, H., Lindberg, G., Hammarlund, B., Palmgren, A. C., Einarsson, C. \& Nord, C. E. (2003). The micro-flora of the small bowel in health and disease. Anaerobe 9, 11-14.

Talavera, G. \& Castresana, J. (2007). Improvement of phylogenies after removing divergent and ambiguously aligned blocks from protein sequence alignments. Syst Biol 56, 564-577.

Tamura, K., Peterson, D., Peterson, N., Stecher, G., Nei, M. \& Kumar, S. (2011). MEGA5: molecular evolutionary genetics analysis using maximum likelihood, evolutionary distance, and maximum parsimony methods. Mol Biol Evol 28, 2731-2739.

Tang, B. S., Lau, S. K., Teng, J. L., Chan, T. M., Chan, W. S., Wong, T. Y., Tong, Y. T., Fan, R. Y., Yuen, K. Y. \& Woo, P. C. (2013). Matrix-assisted laser desorption ionisation-time of flight mass spectrometry for rapid identification of Laribacter hongkongensis. J Clin Pathol 66, 1081-1083. 